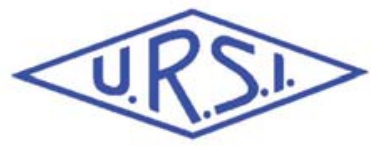

\title{
A Study and analysis of stratum 1 set up establishment at NIC-NKN Delhi for IST synchronization of NKN network
}

\author{
Pranalee P. Thorat*, Preeti Khandpal, and Trilok Bhardwaj \\ Time \& Frequency Division, CSIR-National Physical Laboratory, New Delhi-12, e-mail: \\ thoratpp@nplindia.org; pranuthorat@gmail.com
}

CSIR- National Physical Laboratory (CSIR-NPL) has set up stratum 1 services at two locations of NIC- NKN. This paper explains the study and analysis of stratum 1 set up establishment at NIC-NKN Delhi for IST synchronization of NKN network

The NIC managed National Knowledge network is a project of government of India to provide high bandwidth internet connectivity to research organizations, government offices, ministries, academic institutions etc. But in order to have stable, smooth and accurate functionality of this giant, state of the art, gigabyte network; it needs to be synchronized in time to a stable and accurate standard reference source. Synchronizing this network throughout the India is the next crucial requirement for the perfect operational assurance. CSIR- NPL is custodian of Indian Standard Time (IST).Time scale of NPL, known as UTC(NPLI) is being maintained with the help of cesium clock and active hydrogen maser. The traceability of our time scale with BIPM is at the level of few nano-seconds (ns).

Looking at the requirement of NKN network, NPL proposes to station a NPL Controlled Remote Oscillator System at NIC(at NIC, New Delhi centre and also another unit to be placed at NIC, Hyderabad to add redundancy). A time standard of uncertainty better than $10^{-10}$ (which is assumed to be sufficient requirement of NIC) at the site of NIC which will be physically connected to NTP server. Hence NIC will also become a Stratum 1 server.

The NTP server was synchronized to $10 \mathrm{MHz}$ output of Rb Frequency standard. This Rb was pre-calibrated at CSIR-NPL. And the network of NIC-NKN is then synchronized to NTP server. Following figure shows the stratum 1 establishment at NIC-NKN Shastri Park.

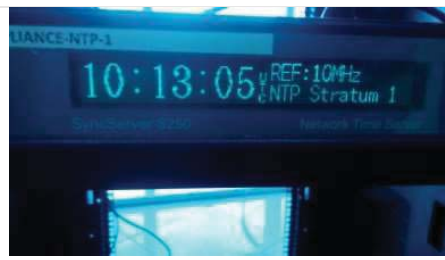

Figure 1: Stratum 1 NTP server installed at NIC-NKN

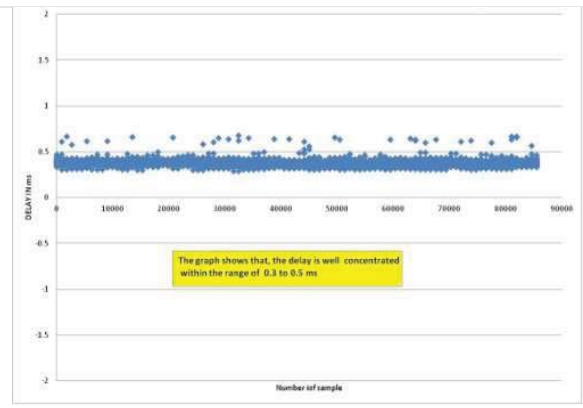

Figure 2: Delay observed in NTP data

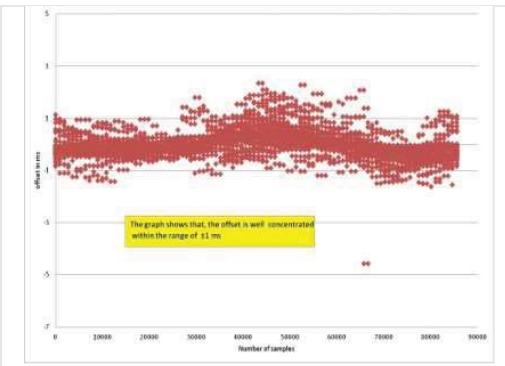

Figure 3: Offset observed in NTP data

This is a first time proof of concept for setting up stratum 1 center in order to have synchronous time PAN India. The results are extremely encouraging as the delay observed is well within the range of 0.3 to $0.5 \mathrm{~ms}$.

1. David L. Mills (12 December 2010). Computer Network Time Synchronization: The Network Time Protocol. Taylor \& Francis. pp. 12-. ISBN 978-0-8493-5805-0

2. David L. Mills (October 1991). "Intern Time Synchronization: The Network Time Protocol" (PDF). IEEE Transactions on Communications. IEEE. 39 (10). doi:10.1109/26.103043. 